\title{
METODOLOGIA PARA ANÁLISE DE SISTEMAS ELÉTRICOS A N CONDUTORES PELO MÉTODO DE INJEÇÃO DE CORRENTES
}

\author{
Débora Rosana Ribeiro Penido*
}

Sandoval Carneiro Jr. ${ }^{\dagger}$

\author{
Leandro Ramos de Araujo*
}

José Luiz Rezende Pereira*

*Depto. de Energia Elétrica, Faculdade de Engenharia, Universidade Federal de Juiz de Fora, Juiz de Fora, MG, Brasil

${ }^{\dagger}$ COPPE, Universidade Federal do Rio de Janeiro,

Caixa Postal 68504, Rio de Janeiro, RJ, Brasil

\section{RESUMO}

Neste trabalho apresenta-se uma nova metodologia para análise em regime permanente de sistemas elétricos a $n$ condutores, com capacidade de modelar diversas características de redes e componentes encontrados nos sistemas elétricos de potência. A metodologia tem se mostrado eficiente e robusta, possuindo aplicação bastante geral, podendo ser utilizada para analisar sistemas equilibrados ou desequilibrados, radiais ou reticulados, de transmissão, subtransmissão, distribuição ou industriais, inclusive sistemas de grande porte. A metodologia proposta foi chamada de Método de Injeção de Correntes a N Condutores - MICN e é baseada no Método de Injeção de Correntes em coordenadas retangulares, definida diretamente em coordenadas de fase e usa o método de Newton-Raphson no processo de solução.

PALAVRAS-CHAVE: Fluxo de potência multifásico, método de injeção de correntes, modelagem de equipamentos, sistemas desequilibrados.

\section{ABSTRACT}

Methodology for Analysis of N-Conductor Electrical Sys-

Artigo submetido em 22/04/2009 (Id.: 00996)

Revisado em 13/07/2009, 18/11/2009

Aceito sob recomendação do Editor Associado Prof. Eduardo N. Asada tems Using the Current Injection Method

In this work a new methodology for steady state analysis of $\mathrm{n}$-conductor electrical systems is presented, with the capability to model a variety of components found in electrical power systems. The methodology is shown to be efficient and robust, with general application and it can be used to analyze balanced or unbalanced systems, radial or meshed, of transmission, sub-transmission, distribution or industrial, including large-scale power systems. The proposed methodology was called the N Conductor Current Injection Method NCIM and is based on the Current Injection Method in rectangular coordinates, formulated directly in phase coordinates and uses the Newton-Raphson method as a solution process.

KEYWORDS: Multi-phase power flow, current injection method, component modeling, unbalanced systems

\section{INTRODUÇÃO}

Atualmente o interesse em modelar os sistemas elétricos com mais detalhes para realizar análises mais precisas tem crescido entre os especialistas em sistemas de potência. Dentre as necessidades atuais destaca-se a modelagem de redes multifásicas desequilibradas.

A maioria das metodologias existentes para solução de sistemas elétricos considera algumas simplificações e adota a 
representação apenas da seqüência positiva das redes ou de equivalente monofásico na modelagem. Os resultados obtidos a partir destas metodologias têm sido utilizados e são suficientes para muitos estudos. Porém, existem situações nas quais a representação equilibrada não é suficientemente precisa, pode-se citar como exemplos: a análise dos sistemas de distribuição, os quais em sua maioria apresentam grandes desequilíbrios entre fases, e a análise de casos de transmissão quando existe um grande desbalanço eletromagnético devido a uma não-transposição de fases ou mesmo linhas de potência natural elevada (LPNE) (Gomes et al., 2002). Além disso, a solicitação por estudos mais detalhados ou de situações particulares tem crescido bastante, especialmente em consequiência do grande incremento da geração distribuída nos sistemas.

Existem vários métodos para solução de sistemas elétricos, principalmente para cálculo de fluxo de potência. Métodos de solução baseados no Método de Newton-Raphson são bastante utilizados, sendo a formulação mais comum em coordenadas polares. Existem diversas variações deste método, sendo que uma metodologia que utiliza otimização de passo pode ser vista no trabalho de Iwamoto e Tamura (1981), a qual foi aprimorada no trabalho de Castro e Braz (1997).

Rotinas para solução do fluxo de potência em sistemas puramente radiais foram propostas em Baran e Wu (1989) e em Céspedes (1990). Para a solução do fluxo de potência trifásico ressaltam-se os trabalhos de Luo e Semlyen (1990) e Cheng e Shirmohammadi (1995) que são baseados em métodos tipo Forward/Backward Sweep (FBS). O FBS tem sido preferido pela maioria dos autores para a solução de sistemas elétricos radiais ou fracamente malhados por possuir nestes casos um bom desempenho e ser de simples implementação computacional. Em Ciric et al. (2003) um método FBS foi expandido para ser utilizado em sistemas a quatro condutores, possibilitando a representação explícita de cabos neutros e aterramentos. Porém, o FBS apresenta dificuldades e até mesmo limitações na presença de redes com dispositivos de controle ou malhadas.

E constantemente muitos outros métodos são propostos, trazendo inovações, que podem ser vistas, por exemplo, nos seguintes trabalhos recentemente publicados. Em Issicaba et al. (2008) apresentou-se uma variação do método de soma das potências para análise de redes de distribuição radiais, denominado Método de Soma das Potências com Rotações. Em Haffner et al. (2008) apresentou-se um modelo aproximado para representar as equações que descrevem os fluxos de potência e tensões em redes de distribuição, sendo as equações aproximadas por modelos lineares. Uma formulação híbrida para fluxo de potência foi proposta em Marinho e Taranto (2008), sendo que a modelagem de sequiência positiva é considerada na transmissão e a modelagem trifásica é considerada na distribuição, conectadas por uma interface passiva, realizando algumas aproximações.

Embora existam muitos métodos para solução de sistemas, atualmente ainda são poucos os que são capazes de resolver algumas situações típicas encontradas em redes reais, como por exemplo, transposições de linhas, grandes razões R/X na distribuição, desbalanço de cargas, configurações mais complexas de topologia e de equipamentos freqüentemente encontradas em sistemas de distribuição, cargas fase-fase, faseneutro, circuitos monofásicos, bifásicos, trifásicos ou com mais condutores, representação explícita de condutores neutros, representação dos aterramentos e solução de sistemas de distribuição muito malhados. Destaca-se inclusive que muitos dos métodos destinados a análises da distribuição aproximam estes sistemas por redes equilibradas. Nota-se então que ainda existem dificuldades ou limitações para muitas metodologias.

Dentre as metodologias de análise existentes, uma linha de pesquisa é baseada no Método de Injeção de Correntes, o qual é baseado nas equações de injeções de correntes escritas em coordenadas retangulares e utiliza o método de Newton-Raphson no processo de solução. Inicialmente foi proposta uma formulação para análise de sistemas de transmissão (Costa et al., 1999). Vislumbrando a utilização em sistemas elétricos trifásicos foi criado o Método de Injeção de Correntes Trifásico (MICT) (Garcia et al., 2001a). Outros desenvolvimentos foram realizados para representação de controles (Garcia et al., 2001b) e melhorias no processo de solução (Araujo, 2000). Para permitir a representação explícita de condutores neutros e aterramentos foi desenvolvido o Método de Injeção de Correntes a Quatro Condutores (MICQ) (Penido et al., 2008). Apesar do MICQ apresentar características interessantes, verificou-se que este método não era realmente geral, pois existiam vários sistemas que não podiam ser simulados por ele. Suas dificuldades e limitações recaiam principalmente em sua estrutura blocada, a qual aumentava muito a dimensão do sistema a ser solucionado e podia dificultar a convergência devido ao tratamento matemático necessário. E ainda, com o MICQ não se conseguia representar componentes que precisassem no modelo de mais de quatro nós na mesma barra.

A partir das limitações do MICQ, e da atual e crescente procura por metodologias mais robustas, que não apresentem as dificuldades de simulação e limitações que ocorrem para vários métodos, decidiu-se desenvolver uma nova metodologia. Esta metodologia mais geral desenvolvida para simular qualquer tipo de sistema foi denominada Método de Injeção de Correntes a N Condutores (MICN). O método proposto possui todas as características interessantes do MICQ (Penido et al., 2008), mas é bem mais flexível, sem a estrutura fixa de blocos, para solucionar sistemas com equipamentos com 
"n" condutores, e pode ser utilizado, com o mesmo código, para sistemas monofásicos, bifásicos, trifásicos, multifásicos, equilibrados ou desequilibrados, radiais ou reticulados, de transmissão, substransmissão, distribuição ou industriais.

Neste trabalho apresenta-se como contribuições a nova metodologia proposta, além de melhorias e desenvolvimentos para modelos de componentes.

\section{MÉTODO DE INJEÇÃO DE CORREN- TES A N CONDUTORES}

\subsection{Fundamentos}

O MICN foi desenvolvido com o intuito de ser uma metodologia para análise de qualquer sistema elétrico, com $n$ condutores. Para isso, todos os nós do sistema são tratados de forma individualizada e os modelos não geram blocos de dimensões pré-definidas, isto contribui para a otimização do processo de solução já que o sistema a ser resolvido pelo método proposto terá conseqüentemente a dimensão estritamente necessária. Esta nova estrutura, mais livre, facilita a formação dos modelos, especialmente de equipamentos com configurações não convencionais.

O MICN baseia-se no método de injeção de correntes e analisando-se suas equações pode-se considerar que em cada nó o somatório de correntes injetadas é formado por parcelas relativas a todos os elementos conectados a ele, e assim pode-se considerar que cada elemento do sistema determina contribuições de injeções de corrente para os nós nos quais ele está conectado. Considere um elemento como uma estrutura conectada entre dois nós.

Seja, por exemplo, um elemento genérico conectado entre os nós $k$ e $m$ do sistema, que tem impedância $z_{k m}$, e admitância $y_{k m}$ igual a $1 / z_{k m}$. Segundo as considerações anteriores este elemento gera uma contribuição de injeção de corrente para o nó $k$ e uma para o nó $m$, que podem ser definidas pelas equações (1) e (2) respectivamente.

$$
\begin{aligned}
& I_{k m}=y_{k m}\left(V_{k}-V_{m}\right) \\
& I_{m k}=y_{k m}\left(V_{m}-V_{k}\right)
\end{aligned}
$$

Assim, no MICN as equações de injeção de corrente vão sendo formadas a partir das contribuições de cada elemento que está ligado em um ou mais nós, sendo que cada elemento é considerado uma unidade básica. Portanto, os modelos da maioria dos componentes (equipamentos do sistema) são na verdade formados por um ou mais elementos conectados nas mais diversas configurações (por exemplo, em estrela ou delta em componentes trifásicos, entre fases, entre fase e neutro, entre fase e terra). A determinação das fases nos nós é feita a partir das ligações com os outros componentes do sistema. Portanto, para a metodologia proposta um nó é apenas um ponto de conexão entre dois elementos, independente da fase.

Em algumas situações utiliza-se a idéia de componente multifásico, pois o mesmo pode apresentar contribuições de injeções de correntes para $n$ nós; isto ocorre, por exemplo, quando se modelam linhas considerando-se as impedâncias mútuas entre fases.

Os dispositivos de controles são normalmente definidos por equações adicionais, que são determinadas de forma a representar uma característica ou função. Estas equações podem ser funções de variáveis relativas aos próprios componentes bem como de outras variáveis do sistema.

\subsection{Processo de Solução}

O método de solução proposto consiste em:

1) Construir as equações de injeções de correntes para todos os nós do sistema a partir das contribuições de todos os elementos, e as equações de controle quando existentes, escritas em coordenadas retangulares, formando um sistema de equações não-lineares, que pode ser representado como:

$$
\mathbf{f}(\mathbf{z})=0
$$

Onde, $\mathbf{z}$ são as variáveis de estado (como por exemplo as partes real e imaginária dos fasores das tensões nodais faseterra e outras variáveis de controle) e f são as equações de injeções de correntes ou funções de controle.

2) Solucionar o conjunto de equações não-lineares, encontrando as variáveis de estado do sistema. No caso utiliza-se o Método de Newton-Raphson. A expressão matemática do sistema (3) linearizado, escrito de forma matricial, é dada por (4):

$$
\mathbf{J}(\mathbf{z}) \Delta \mathbf{z}=-\mathbf{f}(\mathbf{z})
$$

Onde, em cada passo do processo iterativo:

$\mathbf{J}(\mathbf{z})$ é a matriz Jacobiana;

$\mathbf{f}(\mathbf{z})$ é o vetor independente, formado pelas equações a serem resolvidas.

$\Delta \mathbf{z}$ é o vetor dos incrementos das variáveis de estado.

Um algoritmo que representa o processo de solução do 


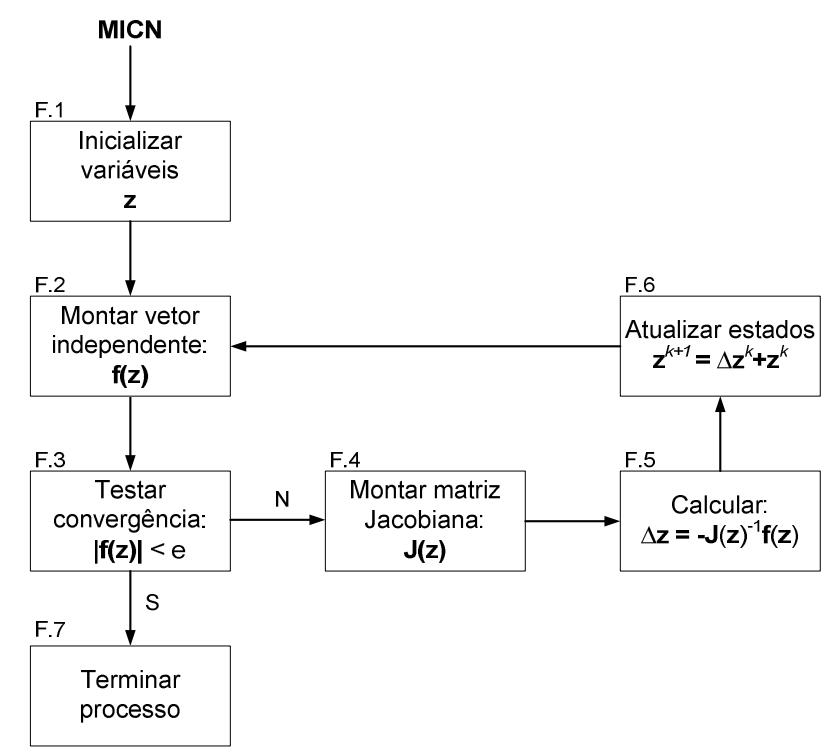

Figura 1: Algoritmo do método de solução do MICN

MICN é apresentado na Figura 1.

\subsection{Inicialização de Variáveis}

A utilização do método de Newton-Raphson para solucionar os sistemas trouxe robustez ao método, o que é muito importante especialmente quando se pretende considerar a representação dos cabos neutros e aterramentos, que é uma modelagem que costuma complicar a convergência da maioria dos métodos (Cheng e Yang, 2001).

O método de Newton-Raphson apresenta propriedade de convergência quadrática perto do ponto de solução, porém necessita de boa inicialização de variáveis antes de seu processo iterativo para se obter sucesso de convergência.

Para a maioria dos sistemas analisados tem sido satisfatória a seguinte inicialização de variáveis no MICN: Tensões das fases - inicializadas com seus valores nominais; Tensões de neutro - feitas inicialmente iguais a zero; Ângulos - em sistemas trifásicos são ajustados usualmente em $0^{\circ},-120^{\circ}$ e $120^{\circ}$ para as fases $a, b$ e $c$, respectivamente; Ângulos de nós de neutros - feitos inicialmente iguais a zero.

\subsection{Aspectos Relevantes}

Neste item resume-se alguns detalhes e aspectos relevantes sobre a metodologia desenvolvida:

- A matriz Jacobiana tem uma grande quantidade de termos nulos ou constantes no MICN, devido à utilização da base do Método de Injeção de Correntes em coorde- nadas retangulares, o que é uma boa característica, uma vez que a ordem do sistema a ser resolvido pode ser elevada, e neste caso torna-se importante a utilização de técnicas de esparsidade para a solução dos sistemas de equações;

- A montagem matricial das equações para o processo de solução é realizada elemento por elemento, de acordo com as suas contribuições e as posições relativas aos nós nos quais está conectado;

- As equações de todos os nós do sistema são construídas, e não se utiliza nenhum artifício para prévia eliminação de equações devido aos "tipos" de barras, por exemplo, barras PV ou V $\theta$;

- As equações são escritas em coordenadas retangulares, utilizando-se como principais variáveis de estado as partes real e imaginária das tensões nodais (fasereferência), além de outras variáveis associadas a controles, e separa-se as equações de injeção de correntes em partes real e imaginária;

- A convergência é verificada diretamente pelos elementos do vetor independente;

- Não há necessidade nesta metodologia de se ter prévia definição se parte da rede ou componente é trifásico, bifásico ou monofásico, ou até possuir mais ramos. A matriz formada para ser utilizada no processo de solução, automaticamente refletirá as características do sistema, ficando com a dimensão estritamente necessária, não havendo necessidade de posterior eliminação de equações relativas a fases inexistentes em determinados componentes monofásicos ou bifásicos, conforme proposto em Garcia et al. (2001a), tornando a metodologia proposta numericamente mais robusta;

- Os componentes podem ser definidos diretamente em coordenadas de fase.

\section{MODELOS PARA O MICN}

\subsection{Base}

De maneira geral, para o MICN é necessário definir para cada componente do sistema elétrico, a partir de seus elementos:

- Suas contribuições de injeções de corrente para todos os nós em que está conectado;

- Suas contribuições para a matriz Jacobiana e para o vetor independente do método de solução de NewtonRaphson, a partir das contribuições de injeções de corrente; 
- E seus dispositivos de controles, se for o caso.

Estas definições formam os modelos e serão apresentadas nas próximas seções para os principais equipamentos dos sistemas elétricos de potência. E os modelos serão definidos com certo grau de detalhamento, de forma a facilitar a compreensão, mas de tal maneira que na prática possam ser simplificados dependendo da necessidade.

Por questões de espaço no artigo apenas as contribuições de injeções de corrente serão apresentadas para todos os equipamentos, exceto para os equipamentos RLC cujas contribuições para o vetor independente e para a Jacobiana também serão apresentados para ilustrar a metodologia proposta.

\subsection{Equipamentos RLC}

Existem diversos equipamentos nos sistemas elétricos que podem ser modelados por elementos contendo apenas resistências, indutâncias e/ou capacitâncias (elementos RLC), conectados das mais diversas maneiras, que serão chamados aqui de componentes/equipamentos RLC. Podemos citar como exemplos os capacitores em derivação para correção de fator de potência, os capacitores série para compensação de reatância de linha e os filtros de harmônicos.

Cada elemento RLC do componente pode ser conectado entre dois nós $(k$ e $m)$ ou entre um nó $(k)$ e a terra. Na primeira possibilidade (Figura 2) o elemento gera contribuições de injeções de corrente para cada nó, as quais são calculadas segundo as equações (5) e (6).

$$
\begin{aligned}
& I_{k m, r l c}=y_{k m}\left(V_{k}-V_{m}\right) \\
& I_{m k, r l c}=y_{k m}\left(V_{m}-V_{k}\right)
\end{aligned}
$$

Caso o elemento RLC esteja conectado entre o nó $k$ e a terra (referência) este gera contribuição de injeção de corrente apenas para o nó $k$ conforme (7).

$$
I_{k, r l c}=y_{k}\left(V_{k}\right)
$$

Para o caso do elemento RLC conectado entre dois nós ( $k$ e $m$ ) este gera contribuições para o vetor independente segundo as equações (8) e (9), e para a matriz Jacobiana segundo (10).

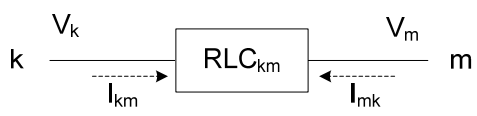

Figura 2: Equipamento RLC

$$
\begin{aligned}
& \mathbf{f}_{k, r l c}(\mathbf{z})=\left[\begin{array}{c}
\operatorname{Im}\left(I_{k m, r l c}\right) \\
\operatorname{Re}\left(I_{k m, r l c}\right)
\end{array}\right]=\left[\begin{array}{c}
\operatorname{Im}\left(y_{k m}\left(V_{k}-V_{m}\right)\right) \\
\operatorname{Re}\left(y_{k m}\left(V_{k}-V_{m}\right)\right)
\end{array}\right] \\
& \mathbf{f}_{m, r l c}(\mathbf{z})=\left[\begin{array}{c}
\operatorname{Im}\left(I_{m k, r l c}\right) \\
\operatorname{Re}\left(I_{m k, r l c}\right)
\end{array}\right]=\left[\begin{array}{c}
\operatorname{Im}\left(y_{k m}\left(V_{m}-V_{k}\right)\right) \\
\operatorname{Re}\left(y_{k m}\left(V_{m}-V_{k}\right)\right)
\end{array}\right]
\end{aligned}
$$

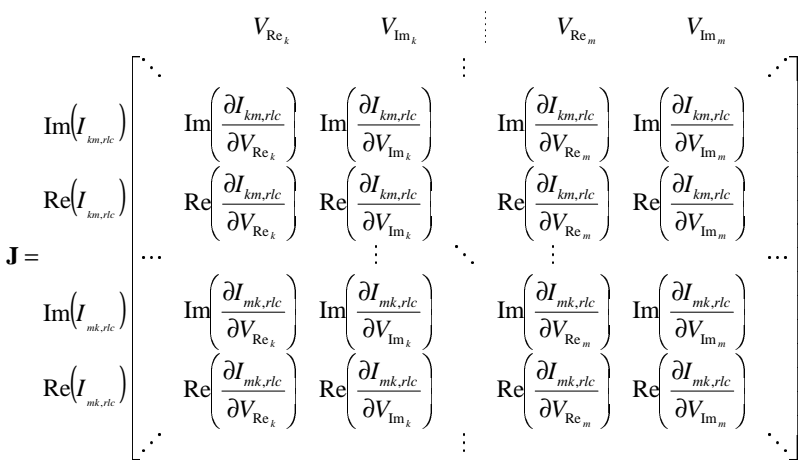

Caso o elemento RLC se encontre conectado entre um nó $k$ e a terra é gerada apenas a contribuição para o vetor independente dada pela equação (11) e a contribuição para a matriz Jacobiana conforme a equação (12).

$$
\begin{aligned}
& \mathbf{f}_{k, r l c}(\mathbf{z})=\left[\begin{array}{c}
\operatorname{Im}\left(I_{k, r l c}\right) \\
\operatorname{Re}\left(I_{k, r l c}\right)
\end{array}\right]=\left[\begin{array}{c}
\operatorname{Im}\left(y_{k}\left(V_{k}\right)\right) \\
\operatorname{Re}\left(y_{k}\left(V_{k}\right)\right)
\end{array}\right]
\end{aligned}
$$

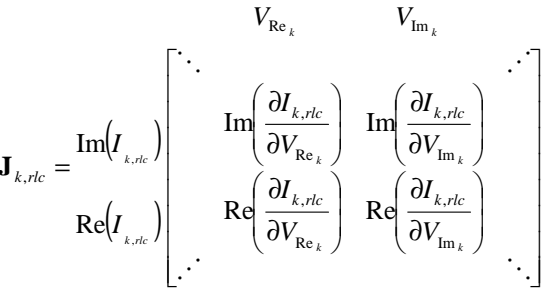

Os elementos RLC do componente podem ser conectados em diversas configurações como, por exemplo, estrela aterrada ou delta formando vários tipos de equipamentos RLC. As contribuições para o vetor independente e para a matriz Jacobiana são feitas a partir de cada elemento.

\subsection{Linhas e Cabos}

O modelo base para linhas e cabos utilizado no MICN é um circuito $\pi$-equivalente a parâmetros concentrados conforme 


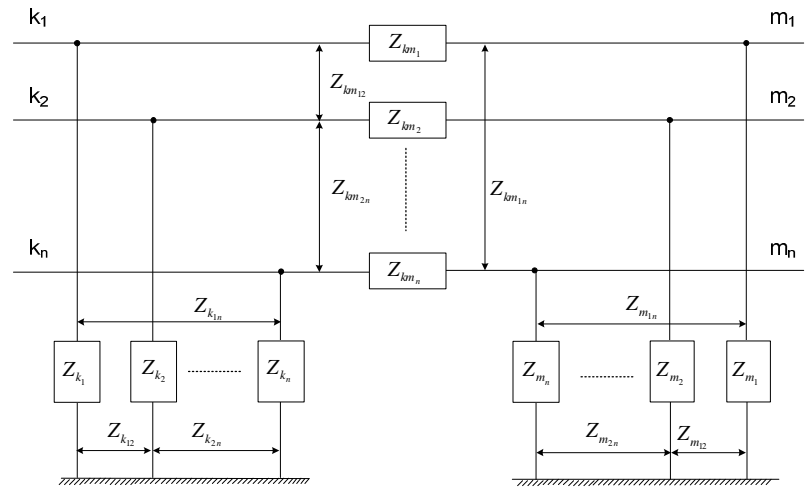

Figura 3: Circuito $\pi$-equivalente

representado de maneira genérica na Figura 3. Os parâmetros das linhas podem ser calculados de diversas maneiras como, por exemplo, em Anderson (1995).

Onde:

$Z_{k m_{x}}$ é a impedância própria entre os nós $k$ e $m$ da fase $x$;

$Z_{k m_{x y}}$ é a impedância mútua entre as fases $x$ e $y$;

$Z_{k_{x}}$ é a impedância em derivação do nó $k$ da fase $x$;

$Z_{k_{x y}}$ é a impedância em derivação mútua entre os nós $k$ da fase $x$ e $k$ da fase $y$.

No MICN, quando as impedâncias mútuas são consideradas, é necessário inicialmente construir a matriz de admitância nodal da linha. Após obter esta matriz, as contribuições da linha para as equações de injeções de corrente de cada nó podem ser escritas separadamente, elemento por elemento da matriz de admitâncias, e sem a necessidade de serem escritas em blocos matriciais, como proposto em Garcia et al. (2001a) e Penido et al. (2008). Porém, apenas para facilitar o entendimento, nas equações (13) e (14) são apresentadas equações matriciais que representam a combinação de todas as contribuições de injeções de correntes nos nós das barras $k$ e $m$ de uma linha genérica (Figura 3) obtidas a partir dos elementos de sua matriz admitância nodal.

$$
\begin{aligned}
& \mathbf{I}_{k m, l i n}=\left[\begin{array}{cccc}
Z_{k m_{1}} & Z_{k m_{12}} & \cdots & Z_{k m_{1 n}} \\
Z_{k m_{21}} & Z_{k m_{2}} & & Z_{k m_{2 n}} \\
\vdots & & \ddots & \\
Z_{k m_{n 1}} & Z_{k m_{n 2}} & & Z_{k m_{n}}
\end{array}\right]^{-1} . \\
& {\left[\begin{array}{c}
V_{k_{1}}-V_{m_{1}} \\
V_{k_{2}}-V_{m_{2}} \\
\vdots \\
V_{k_{n}}-V_{m_{n}}
\end{array}\right]+} \\
& +\left[\begin{array}{cccc}
Z_{k_{1}} & Z_{k_{12}} & \cdots & Z_{k_{1 n}} \\
Z_{k_{21}} & Z_{k_{2}} & & Z_{k_{2 n}} \\
\vdots & & \ddots & \\
Z_{k_{n 1}} & Z_{k_{n 2}} & & Z_{k_{n}}
\end{array}\right]^{-1}\left[\begin{array}{c}
V_{k_{1}} \\
V_{k_{2}} \\
\vdots \\
V_{k_{n}}
\end{array}\right] \\
& \mathbf{I}_{m k, l i n}=\left[\begin{array}{cccc}
Z_{k m_{1}} & Z_{k m_{12}} & \cdots & Z_{k m_{1 n}} \\
Z_{k m_{21}} & Z_{k m_{2}} & & Z_{k m_{2 n}} \\
\vdots & & \ddots & \\
Z_{k m_{n 1}} & Z_{k m_{n 2}} & & Z_{k m_{n}}
\end{array}\right]^{-1} . \\
& {\left[\begin{array}{c}
V_{m_{1}}-V_{k_{1}} \\
V_{m_{2}}-V_{k_{2}} \\
\vdots \\
V_{m_{n}}-V_{k_{n}}
\end{array}\right]+} \\
& +\left[\begin{array}{cccc}
Z_{m_{1}} & Z_{m_{12}} & \cdots & Z_{m_{1 n}} \\
Z_{m_{21}} & Z_{m_{2}} & & Z_{m_{2 n}} \\
\vdots & & \ddots & \\
Z_{m_{n 1}} & Z_{m_{n 2}} & & Z_{m_{n}}
\end{array}\right]^{-1}\left[\begin{array}{c}
V_{m_{1}} \\
V_{m_{2}} \\
\vdots \\
V_{m_{n}}
\end{array}\right]
\end{aligned}
$$

A partir dos elementos deste modelo é possível representar linhas com várias características, como parâmetros assimétricos, cabos pára-raios, cabos neutros, acoplamentos entre linhas próximas, ramais monofásicos, bifásicos e linhas multifásicas, importantes especialmente para sistemas de distribuição (IEEE Test Feeders, online) bem como para análise de sistemas elétricos contendo LPNE.

\subsection{Cabos Neutros e Aterramentos}

Em muitas das metodologias atuais de solução de sistemas, particularmente na maioria das ferramentas de solução de fluxo de potência, ao simular linhas com cabos neutros, estes são desconsiderados para simplificar a modelagem. Entretanto, considera-se que modelar os cabos neutros e os aterramentos pode ser muito importante, para poder conhecer as grandezas elétricas dos mesmos e para determinar corretamente os desequilíbrios. 


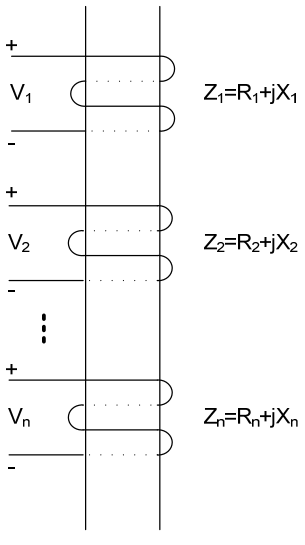

Figura 4: Modelo do transformador genérico

No MICN o cabo neutro quando existente pode ser explicitamente representado, modelado como se fosse qualquer outra fase da linha, sem precisar de equacionamento diferente para neutro ou de cálculos adicionais ao fim do processo iterativo para determinar suas grandezas. Adicionalmente, qualquer aterramento existente pode ser modelado como um equipamento RLC, utilizando-se as equações já apresentadas na seção 3.2 .

\subsection{Transformadores}

A modelagem de transformadores do MICN permite representar qualquer combinação de ligações de transformadores de $n$ enrolamentos, como por exemplo, as conexões básicas estrela (Y) ou delta $(\Delta)$, além de outros tipos especiais de conexão. Este modelo foi desenvolvido tendo como base o que está apresentado em Anderson (1995) e Elgerd (1977).

A Figura 4 apresenta o modelo de um transformador genérico constituido de um único nucleo, com $\mathrm{n}$ enrolamentos.

Para modelar um transformador no MICN deve-se inicialmente determinar quantos transformadores genéricos são necessários e como conectar os enrolamentos de acordo com o transformador que se deseja representar. Após esta determinação deve-se montar a matriz admitância nodal de barras para cada um dos transformadores genéricos. Na Figura 5 apresenta-se o algoritmo utilizado para a montagem da matriz admitância nodal de barras para um transformador genérico de $n$ enrolamentos, que é a base do modelo do MICN.

Na seqüência o que é feito para representar os transformadores no MICN assemelha-se ao que é feito para modelar as linhas. Para cada transformador genérico, após obter a matriz admitância nodal, as contribuições deste transformador genérico para as equações de injeções de corrente de cada nó podem ser escritas separadamente, elemento por elemento da

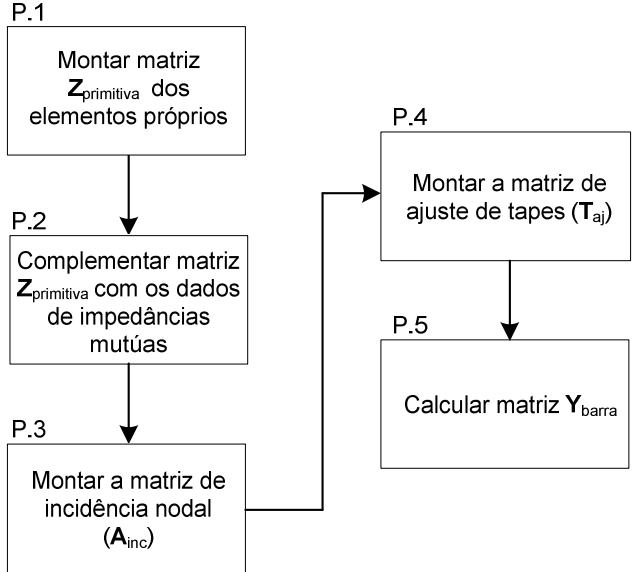

Figura 5: Algoritmo de montagem da matriz $Y_{\text {barra }}$ de transformador genérico

matriz, independentemente de ordem de fases ou nós, e sem a necessidade de serem escritas em blocos matriciais. E finalmente, as contribuições de todos os elementos, de todas as matrizes de admitância nodal, de todos os transformadores genéricos utilizados, quando combinadas, considerandose os nós de conexão dos enrolamentos dos transformadores genéricos, formam o modelo completo do transformador do sistema.

\subsection{Cargas}

As cargas conectadas a um sistema elétrico de potência apresentam-se na realidade nas mais diversas formas como, por exemplo, motores, iluminação, etc. Estes componentes são normalmente modelados como cargas equivalentes conectadas às barras do sistema, sendo que a potência consumida por eles pode variar conforme o seu tipo. São vários os modelos de carga tratados na literatura, e para métodos de solução de fluxo de potência os mais empregados são o ZIP e o exponencial.

No MICN utiliza-se o modelo de carga exponencial, o qual permite a representação de cargas com várias características, inclusive os tipos potência constante, corrente constante e impedância constante. E cada carga é composta por combinação de elementos, representados esquematicamente na Figura 6, onde para cada elemento carga: $S_{0}=P_{0}+j Q_{0}$.

$S_{0}$ é a potência aparente; $P_{0}$ é a potência ativa; $Q_{0}$ é a potência reativa (valores referentes quando uma tensão $V_{0}$ é aplicada aos terminais do elemento).

Quando uma tensão $V_{k m}$ é aplicada na carga, os valores das potências ativa e reativa podem ser obtidos pela expressão (15), sendo $\alpha$ e $\beta$ os coeficientes representativos do compor- 


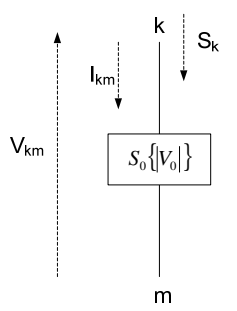

Figura 6: Elemento Carga - Modelo Exponencial

tamento de variação desta carga com a tensão.

$$
S_{k m}=P_{0}\left(\frac{\left|V_{k m}\right|}{\left|V_{0}\right|}\right)^{\alpha}+j Q_{0}\left(\frac{\left|V_{k m}\right|}{\left|V_{0}\right|}\right)^{\beta}
$$

As contribuições de injeções de correntes de um elemento carga conectado entre os nós $k$ e $m$ podem ser calculadas utilizando-se as expressões (16) e (17):

$$
\begin{aligned}
& I_{k m, c r g}=\left(\frac{S_{k m}}{V_{k m}}\right)^{*} \\
& I_{m k, c r g}=\left(\frac{S_{k m}}{V_{m k}}\right)^{*}
\end{aligned}
$$

A metodologia proposta permite representar vários tipos de conexões das cargas, em estrela ou em delta, monofásicas, bifásicas ou trifásicas, tanto equilibradas como desequilibradas.

Em sistemas de distribuição, cargas são freqüentemente encontradas conectadas ao neutro ou entre fases, e nesses casos uma representação de sequiência positiva não funcionaria adequadamente. No MICN, por causa de sua modelagem pelos elementos, as cargas monofásicas e bifásicas são modeladas sem problemas, bastando considerar apenas os elementos das fases existentes. O MICN permite representar também aterramento de neutro de carga, que pode ser feito por meio de um equipamento RLC.

\subsection{Geradores e Barras de Referência}

A modelagem da geração na maioria dos programas de análise de sistemas tem sido feita considerando tipos para suas barras (o que também é feito algumas vezes para cargas), sendo os principais tipos $\mathrm{V} \theta$, PV e PQ. No modelo de gerador desenvolvido para o MICN optou-se por não utilizar este tipo de formulação convencional. O que se faz aqui é escrever as equações de injeções de corrente para todos os nós, de todas as barras, independente de seus tipos. Assim, é possível representar inclusive aterramentos nos geradores quando existentes, ou neutros flutuantes, portanto permitindo que as tensões de neutro dos geradores sejam diferentes de zero dependendo do desequilíbrio. Desta maneira, especialmente para sistemas desequilibrados pode-se ter uma melhor representação. Além disso, como neste método não se elimina equações de nenhum tipo de barra, não é necessário usar rotinas de eliminação de equações nos sistemas e nem realizar pós-processamento para calcular potências das barras (Garcia et al., 2001a). Considera-se esta nova abordagem como uma contribuição do MICN.

Na modelagem do MICN, para representação de geradores com características de barras $\mathrm{V} \theta$ ou $\mathrm{PV}$, ou qualquer outro tipo que não seja PQ, são utilizados controles, ou seja, são introduzidas equações adicionais no sistema de equações nãolineares a ser resolvido e consequentemente são criadas novas variáveis de estado.

Lembra-se que o procedimento básico para incorporar controles na metodologia proposta é utilizar equações adicionais para representar as ações de controle, resultando em um sistema e conseqüientemente em uma matriz Jacobiana estendidos.

É importante destacar também que na realidade a maioria absoluta dos geradores são trifásicos, porém, no método proposto, para efeitos de construção do modelo básico de gerador, pode-se considerar que o gerador trifásico (fonte de tensão) é formado por três elementos monofásicos e utilizar um acoplamento mútuo caso seja de interesse. Isto será feito no equacionamento dos modelos nesta seção, apenas para manter a generalidade da filosofia da modelagem a $n$ condutores, que considera os elementos individualmente.

As contribuições de injeções de correntes de um elemento gerador conectado entre os nós $k$ e $m$ podem ser calculadas utilizando-se as expressões (18) e (19):

$$
\begin{gathered}
I_{k m, g e r}=-\left|V_{k}-V_{m}\right|^{\alpha}\left(\frac{P_{g e r}+j Q_{g e r}}{V_{k}-V_{m}}\right)^{*} \\
I_{m k, g e r}=-\left|V_{m}-V_{k}\right|^{\alpha}\left(\frac{P_{g e r}+j Q_{g e r}}{V_{m}-V_{k}}\right)^{*}
\end{gathered}
$$

Onde $P_{g e r}$ e $Q_{g e r}$ serão valores especificados ou variáveis dependendo de cada caso, os quais serão definidos mais à frente. Normalmente os geradores síncronos são modelados como gerações de potência sem a correção da tensão, portanto nestes modelos os valores de $\alpha$ são definidos como zero.

F.1) Geradores com potência ativa e reativa especificadas (barras tipo PQ)

Neste caso $P_{g e r}$ e $Q_{g e r}$ são valores especificados, ou seja, constantes. 
F.2) Geradores com módulo e ângulo de tensão especificados entre nós (barras tipo $\mathrm{V} \theta$ - barra de referência)

Neste caso, além das contribuições de injeções de correntes, são utilizados controles definidos para fixar a tensão entre dois pontos e o ângulo, o que se traduz na metodologia proposta em duas equações extras, (20) e (21), e conseqüentemente na criação de duas novas variáveis de estado, que no caso utiliza-se: $P_{g e r}$ e $Q_{g e r}$. Lembra-se que estas variáveis terão seus valores atualizados a cada passo do processo iterativo.

$$
\begin{gathered}
\mathbf{f}_{1, g e r}(\mathbf{z})=R e\left(V_{e s p} \mathrm{e}^{j \theta_{e s p}}-V_{k m}\right)=V_{R e_{e s p}}-V_{R e_{k m}} \\
\mathbf{f}_{2, \text { ger }}(\mathbf{z})=\operatorname{Im}\left(V_{e s p} \mathrm{e}^{j \theta_{e s p}}-V_{k m}\right)=V_{I m_{e s p}}-V_{I m_{k m}}
\end{gathered}
$$

Onde, entre os nós $k$ e $m$ :

$V_{e s p} \mathrm{e}^{j \theta_{e s p}}=V_{R e_{e s p}}+j V_{I m_{e s p}}$ é o fasor de tensão especificado;

$V_{k m}=V_{R e_{k m}}+j V_{I m_{k m}}$ é o fasor de tensão calculado;

$V_{e s p}$ é o módulo do fasor de tensão especificado;

$\theta_{\text {esp }}$ é o ângulo do fasor de tensão especificado.

F.3) Geradores com potência ativa e módulo de tensão entre nós especificados (barras tipo PV)

Para representar os elementos dos geradores com características de potência ativa e módulo de tensão especificados o tratamento é análogo àquele dado aos elementos das barras tipo $\mathrm{V} \theta$. Neste caso adiciona-se uma equação extra de controle para fixar o módulo da tensão entre os dois pontos em um valor especificado (22), e consequentemente cria-se uma nova variável de estado, no caso $Q_{g e r}$ Neste caso $P_{g e r}$ é um valor constante, especificado.

$$
\mathbf{f}_{1, g e r}(\mathbf{z})=\left[V_{e s p}^{2}-\left|V_{k m}\right|^{2}\right]=\left[V_{e s p}^{2}-V_{R e_{k m}}^{2}-V_{I m_{k m}}^{2}\right]
$$

\section{EXEMPLOS DE APLICAÇÕES}

Dentre os sistemas utilizados para testar a metodologia proposta encontram-se os sistemas teste de distribuição do DSASC da PES/IEEE (IEEE Test Feeders, online), que são desenvolvidos com o intuito de formarem uma base para validação de metodologias para análise de sistemas de distribuição e de teste de modelos. Recentemente alguns sistemas testes foram criados ou modificados para incluir novos tipos de transformadores, configurações mais complexas, circuitos neutros e aterramentos, como desafios de representação para as metodologias. Todos os sistemas teste de distribuição, com todos os seus detalhes, estão sendo representados e simulados corretamente pelo MICN, reproduzindo todos os resultados disponibilizados pelo subcomitê, validando a metodologia proposta e mostrando principalmente sua flexibilidade. Além disso, vários outros sistemas têm sido utilizados para testar o MICN, sendo que o método tem se mostrado eficiente e preciso, além de muito robusto, uma vez que a convergência é normalmente alcançada com poucas iterações.

Nesta seção serão apresentados alguns resultados obtidos a partir da simulação de 3 sistemas com o MICN, procurando mostrar algumas das capacidades da metodologia proposta.

\subsection{Linha Circuito Duplo}

O objetivo principal deste exemplo é mostrar a flexibilidade do método proposto, uma vez que este permite simular os sistemas considerando seus vários modelos, por exemplo, utilizando o monofásico equivalente, com sua representação trifásica ou representando seus $n$ condutores. Isto pode ser feito naturalmente no MICN sem a necessidade de realizar ajustes na metodologia ou no programa computacional. Esta característica torna a ferramenta proposta extremamente geral.

Para apresentar esta característica do método e mostrar os resultados que se pode obter, além de suas diferenças dependendo do nível de detalhes considerado na modelagem, foi criado um sistema bastante simples. Este sistema consiste em uma linha de circuito duplo de $345 \mathrm{kV}$ (Figura 7) com dois cabos de guarda (PR). Os dados desta linha estão apresentados em Anderson (1995). Definiu-se o comprimento de $300 \mathrm{~km}$ para a linha e aterramento dos cabos de guarda por resistências de 10 ohms. Os efeitos das capacitâncias não foram considerados, semelhantemente ao realizado em Anderson (1995). Uma carga trifásica equilibrada de 300 MVA, com fator de potência igual a 0,98 , potência constante, foi conectada em uma extremidade da linha e na outra extremidade as tensões foram mantidas fixas e equilibradas nos valores nominais. O diagrama unifilar do sistema proposto é apresentado na Figura 8. Foram preparados quatro casos a partir deste sistema que são apresentados a seguir:

1f - Sistema representado pelo equivalente monofásico (apenas dados de sequência positiva). Os parâmetros de sequência positiva foram calculados utilizando componentes simétricas.

3f - Sistema representado pelo equivalente trifásico, os efeitos dos cabos de guarda foram incorporados nas fases da linha utilizando redução de Kron. 


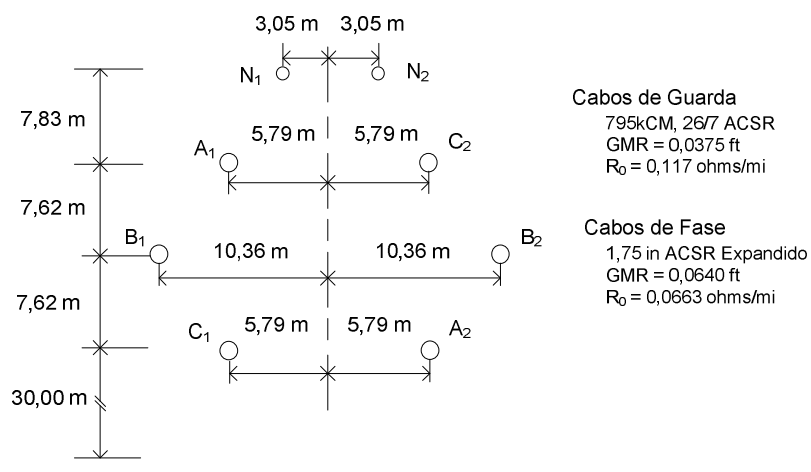

Figura 7: Linha circuito duplo - $345 \mathrm{kV}$

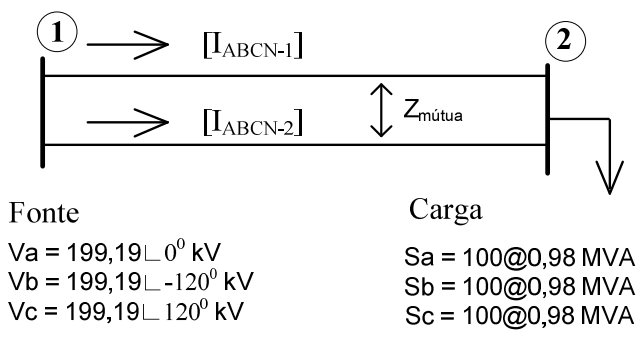

Figura 8: Sistema em $345 \mathrm{kV}$

$4 \mathbf{f}$ - Sistema representado pelo equivalente a quatro condutores. Mas as impedâncias mútuas entre os circuitos não foram representadas neste caso.

8f - Todos os detalhes foram representados. Inclusive impedâncias mútuas entre os circuitos.

Na Tabela 1 são apresentados os resultados das tensões faseterra dos nós da linha de transmissão no lado da carga (barra 2) representados em cada um dos quatro casos. Para cada modelagem considerada na simulação encontra-se seus resultados correspondentes, sendo que algumas grandezas só podem ser verificadas quando modelagens mais completas são consideradas, e além disso, pode-se notar diferenças entre os resultados devido as simplificações efetuadas em alguns dos modelos considerados.

Na Tabela 2 são apresentados os valores dos módulos de correntes nos condutores da linha de transmissão para cada caso simulado. Pode-se verificar os resultados obtidos e suas diferenças, com destaque para os resultados de corrente nos cabos de guarda.

As perdas de potência ativa na linha de transmissão obtidas considerando a modelagem de cada caso são apresentadas na Tabela 3.

Mesmo neste caso bastante simples e pouco desequilibrado pode-se observar quais os resultados que podem ser obtidos
Tabela 1: Tensões fase-terra ( $\mathrm{kV}$ e graus)

\begin{tabular}{|c|c|c|c|c|}
\hline Fase $\mid$ Caso & 1f & $3 \mathrm{f}$ & $4 \mathrm{f}$ & $8 \mathrm{f}$ \\
\hline \multirow{2}{*}{$\mathrm{A}_{1}$} & 184,77 & 180,86 & 180,78 & 183,51 \\
& $-10,7$ & $-10,93$ & $-10,94$ & $-10,24$ \\
\hline \multirow{2}{*}{$\mathrm{B}_{1}$} & - & 185,70 & 185,73 & 185,38 \\
& & $-130,55$ & $-130,56$ & $-130,50$ \\
\hline \multirow{2}{*}{$\mathrm{C}_{1}$} & - & 187,88 & 187,86 & 187,19 \\
& & 109,40 & 109,41 & 109,91 \\
\hline \multirow{2}{*}{$\mathrm{A}_{2}$} & 184,77 & 180,86 & 180,78 & 183,51 \\
& $-10,7$ & $-10,93$ & $-10,94$ & $-10,24$ \\
\hline \multirow{2}{*}{$\mathrm{B}_{2}$} & - & 185,70 & 185,73 & 185,38 \\
& & $-130,55$ & $-130,56$ & $-130,50$ \\
\hline \multirow{2}{*}{$\mathrm{C}_{2}$} & - & 187,88 & 187,86 & 187,19 \\
& & 109,40 & 109,41 & 109,91 \\
\hline \multirow{2}{*}{$\mathrm{N}_{1}$} & - & - & 0,311 & 0,136 \\
& & - & 0,239 & 0,102 \\
\multirow{2}{*}{$\mathrm{N}_{2}$} & - & - & $-49,08$ & $-68,56$ \\
\hline
\end{tabular}

Tabela 2: Corrente nos condutores (A)

\begin{tabular}{|c|c|c|c|c|}
\hline Fase $\mid$ Caso & $1 \mathrm{f}$ & $3 \mathrm{f}$ & $4 \mathrm{f}$ & $8 \mathrm{f}$ \\
\hline $\mathrm{A}_{1}$ & 270,60 & 282,582 & 282,819 & 273,110 \\
\hline $\mathrm{B}_{1}$ & - & 268,957 & 268,438 & 269,643 \\
\hline $\mathrm{C}_{1}$ & - & 261,013 & 261,474 & 266,507 \\
\hline $\mathrm{A}_{2}$ & 270,60 & 270,330 & 270,345 & 271,819 \\
\hline $\mathrm{B}_{2}$ & - & 272,301 & 270,039 & 269,788 \\
\hline $\mathrm{C}_{2}$ & - & 265,801 & 270,862 & 267,683 \\
\hline $\mathrm{N}_{1}$ & - & - & 31,175 & 13,678 \\
\hline $\mathrm{N}_{2}$ & - & - & 23,959 & 10,281 \\
\hline
\end{tabular}

em cada situação de modelagem, além das diferenças numéricas entre os resultados de mesmas grandezas, dependendo da modelagem considerada. É preciso ressaltar que os erros em resultados, oriundos de simplificações adotadas nas modelagens de sistemas tornam-se maiores quanto maiores e mais desequilíbrados forem os sistemas, o que necessita atenção especial dependendo do estudo a ser realizado.

Tabela 3: Perdas ativas (kW)

\begin{tabular}{|c|c|c|c|c|}
\hline Caso & $1 \mathrm{f}$ & $3 \mathrm{f}$ & $4 \mathrm{f}$ & $8 \mathrm{f}$ \\
\hline Perdas & 5467,8 & 5472,9 & 5475,8 & 5407,1 \\
\hline
\end{tabular}


A ferramenta proposta permite representar os sistemas com tantos detalhes seja desejável, com grande precisão e desempenho computacional. O MICN permite representar de forma eficiente, sem artifícios computacionais, inclusive as impedâncias mútuas entre condutores, mesmo em diferentes níveis de tensão.

\subsection{Caso IEEE4 Modificado}

No caso teste apresentado na Figura 9 (IEEE Test Feeders, online) o transformador possui uma conexão para a terra (tape) no meio de um enrolamento do delta do secundário. De acordo com Dugan (2004) esta é considerada uma das conexões de transformador que são encontradas em sistemas da distribuição mais complicadas de se modelar e representa um desafio adicional para os desenvolvedores de metodologias de análise de sistemas de distribuição.

O tape central ("mid tap") é aterrado, fazendo a referência de tensão do secundário se deslocar para uma posição incomum na análise do circuito trifásico. Dessa forma uma fase tem a tensão significativamente mais elevada em relação à terra do que as outras.

Na Tabela 4 são apresentados os resultados das tensões faseterra dos nós do sistema e na Tabela 5 são apresentadas as correntes nas linhas e cabos. Como se pode observar nas tabelas, a tensão de neutro do lado em Y do transformador é de $\mathrm{V}_{2 t}=63,26 \perp 3,047^{0} \mathrm{~V}$, a qual reflete o deslocamento do neutro devido a operação desbalanceada. Pode-se verificar também a corrente que passa no tape central do lado em delta do transformador que é de $9,86 \perp 129,28^{\circ} \mathrm{A}$.

Ressalta-se que este sistema foi facilmente modelado utilizando-se a metodologia proposta (MICN), mesmo tendo

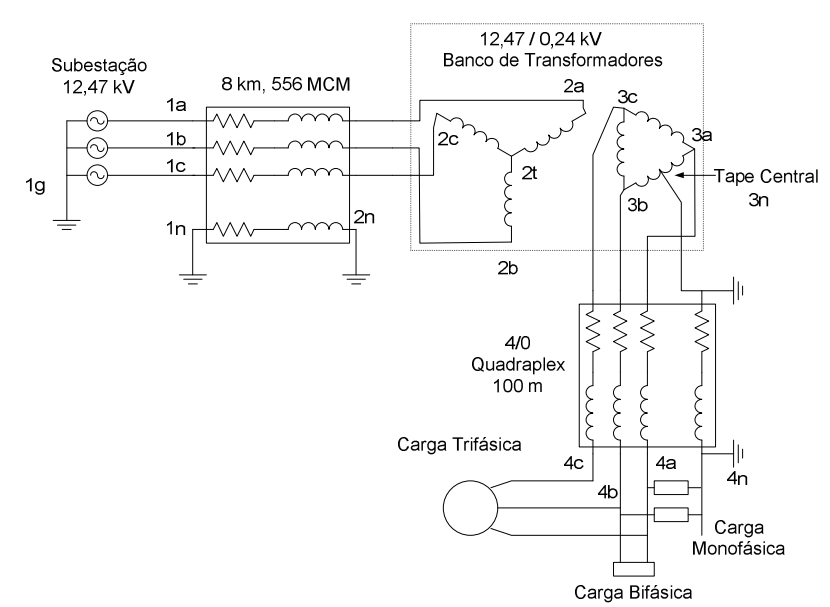

Figura 9: Sistema 4 barras com transformador Y-Delta com tape central
Tabela 4: Tensões nos nós (Fase-Terra)

\begin{tabular}{|c|c|c|}
\hline Nós & Tensão $(\mathrm{kV})$ & Ângulo $\left(^{\circ}\right)$ \\
\hline $1 \mathrm{a}$ & 7,200 & 0,00 \\
\hline $1 \mathrm{~b}$ & 7,200 & $-120,00$ \\
\hline $1 \mathrm{c}$ & 7,200 & 120,00 \\
\hline $1 \mathrm{n}$ & 0,000 & 0,00 \\
\hline $1 \mathrm{~g}$ & 0,000 & 0,00 \\
\hline $2 \mathrm{a}$ & 7,194 & $-0,05$ \\
\hline $2 \mathrm{~b}$ & 7,194 & $-120,01$ \\
\hline $2 \mathrm{c}$ & 7,197 & 119,97 \\
\hline $2 \mathrm{t}$ & 0,063 & 3,047 \\
\hline $2 \mathrm{n}$ & 0,000 & 0,00 \\
\hline $3 \mathrm{a}$ & 117,14 & $-0,51$ \\
\hline $3 \mathrm{~b}$ & 117,00 & 179,52 \\
\hline $3 \mathrm{c}$ & 204,13 & 89,86 \\
\hline $3 \mathrm{n}$ & 0,000 & 0,00 \\
\hline $4 \mathrm{a}$ & 116,25 & $-0,25$ \\
\hline $4 \mathrm{~b}$ & 115,46 & 179,42 \\
\hline $4 \mathrm{c}$ & 203,68 & 89,85 \\
\hline $4 \mathrm{n}$ & 0,000 & 0,00 \\
\hline
\end{tabular}

Tabela 5: Correntes em linhas

\begin{tabular}{|c|c|c|c|}
\hline De & Para & Corrente $(\mathrm{A})$ & Ângulo $\left(^{\circ}\right)$ \\
\hline $1 \mathrm{a}$ & $2 \mathrm{a}$ & 2,747 & $-29,422$ \\
\hline $1 \mathrm{~b}$ & $2 \mathrm{~b}$ & 1,763 & $-175,765$ \\
\hline $1 \mathrm{c}$ & $2 \mathrm{c}$ & 1,610 & 113,217 \\
\hline $1 \mathrm{n}$ & $2 \mathrm{n}$ & 0,040 & $-21,329$ \\
\hline $3 \mathrm{a}$ & $4 \mathrm{a}$ & 114,574 & $-42,435$ \\
\hline $3 \mathrm{~b}$ & $4 \mathrm{~b}$ & 138,048 & 161,349 \\
\hline $3 \mathrm{c}$ & $4 \mathrm{c}$ & 58,893 & 55,092 \\
\hline $3 \mathrm{n}$ & $4 \mathrm{n}$ & 10,787 & $-50,115$ \\
\hline
\end{tabular}

barras com vários nós (exemplo: na barra 2 existem 5 nós), uma conexão de transformador mais complexa, cargas monofásicas, bifásicas e trifásicas, e não apresentou problemas de convergência, convergindo em quatro iterações.

\subsection{Sistema IEEE - NEV Simples}

O objetivo principal deste caso é verificar a capacidade de metodologias de calcular as tensões de neutro para a terra 


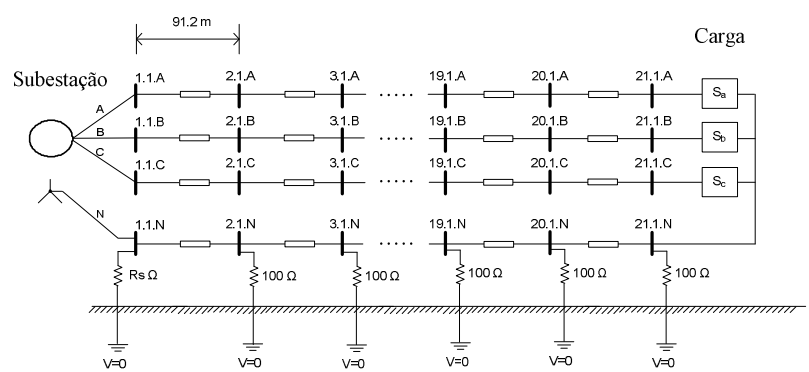

Figura 10: Circuito NEV

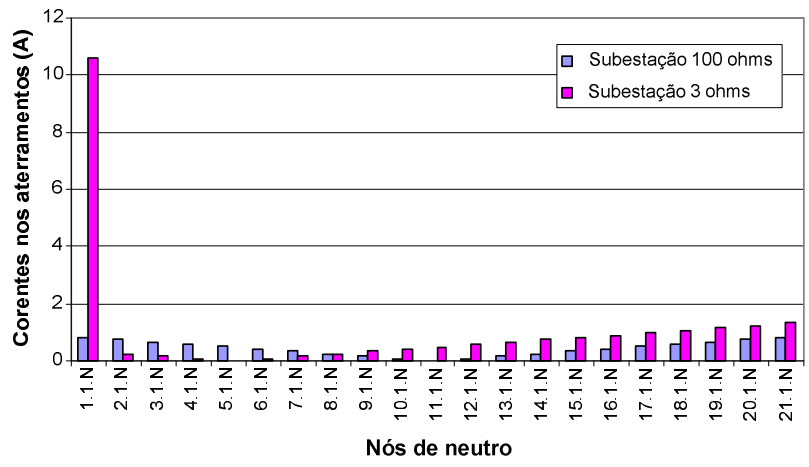

Figura 11: Correntes nos aterramentos ( 3 ohms $\times 100$ ohms)

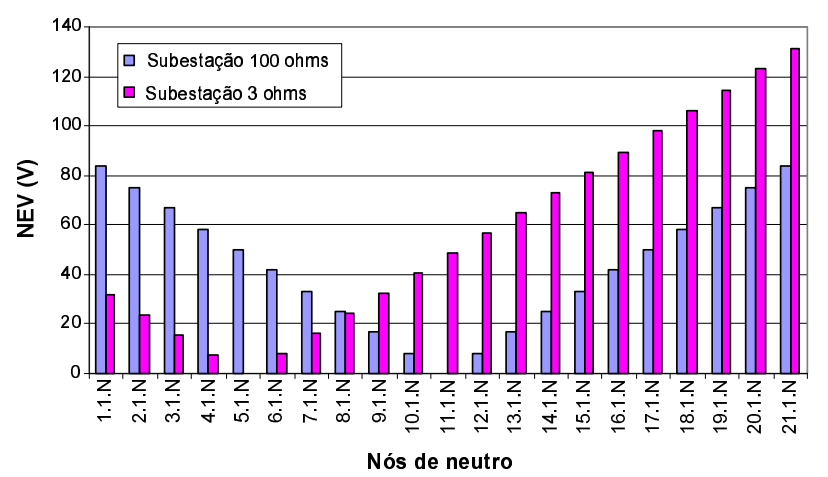

Figura 12: Tensões de neutro (3 ohms $\times 100$ ohms)

o neutro da subestação é aterrado por 3 ohms, porém em outro poste. Estes padrões são encontrados em casos reais e puderam ser verificados pela metodologia proposta, porque a mesma permite representar a subestação e o sistema de forma detalhada.

Na Figura 13 são apresentados os resultados de tensão faseterra para os nós das fases $\mathrm{A}, \mathrm{B}$ e $\mathrm{C}$ do sistema quando a subestação encontra-se aterrada por $100 \mathrm{ohms}$. Verifica-se que a tensão fase-terra da subestação encontra-se desbalanceada devido a tensão não nula do nó de neutro da subestação. Por exemplo, a tensão de neutro-terra da subestação (1.1.N) é $83,8 \perp 151,27^{0} \mathrm{~V}$, a tensão da fase A-neutro é $7200,0 \perp 0,0^{\circ} \mathrm{V}$, $\log$ a tensão fase A-terra é $7126,6 \perp 0,32^{0} \mathrm{~V}$. A fase $\mathrm{C}$ possui um carregamento menor que as fases A e B, logo esta possui uma tensão mais elevada.

Na Figura 14 é apresentada uma comparação entre as tensões fase A-terra quando a subestação encontra-se aterrada por 3 ohms e por $100 \mathrm{ohms}$.

\section{CONCLUSÕES}

Neste trabalho propôs-se um método geral para solução e análise de sistemas elétricos denominado Método de Injeção 


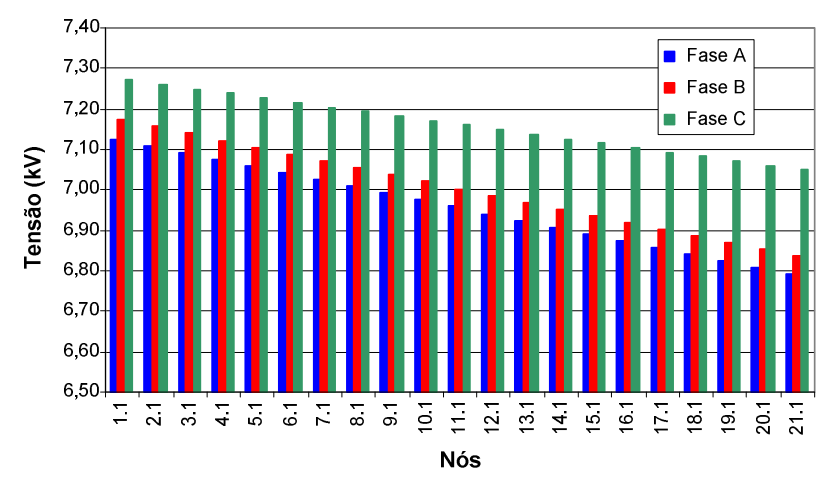

Figura 13: Tensões das fases A, B e C (100 ohms)

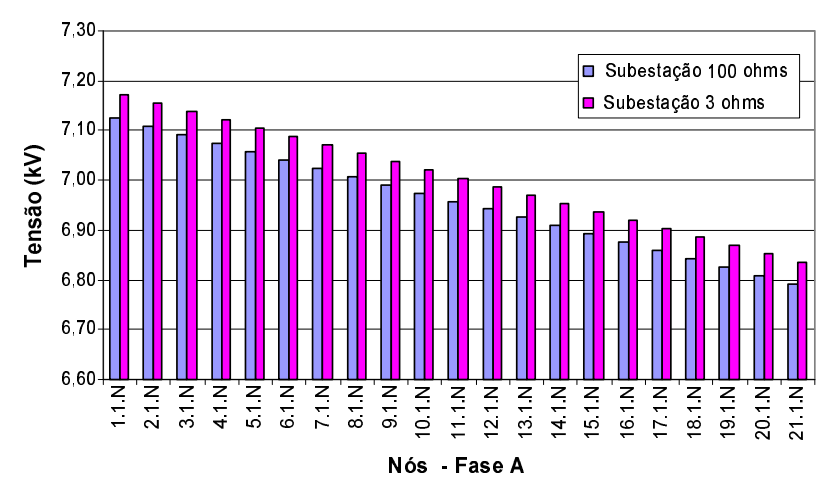

Figura 14: Tensões da fase A ( 3 ohms $\times 100$ ohms)

\section{de Correntes a N Condutores - MICN.}

O desenvolvimento do MICN teve como um dos focos possibilitar a representação dos sistemas com muitos detalhes, sempre que desejável e possível, assim análises mais completas poderiam ser realizadas. Considerou-se a formulação direta em coordenadas de fase e a aplicação do método de injeção de correntes em coordenadas retangulares, aproveitando sua estrutura esparsa semelhante a da matriz admitância nodal.

Criou-se uma modelagem por elementos, na qual os componentes não geram blocos de dimensões pré-definidas, e representam estritamente o necessário. Esta nova estrutura, se mostrou muito flexível, facilitando a formação dos modelos, especialmente de equipamentos com configurações diferentes, que em algumas metodologias anteriores necessitavam de artifícios e simplificações para serem representados, ou até mesmo não podiam ser representados. Por outro lado, para cada componente do sistema, somente os elementos realmente existentes são modelados, fazendo com que as matrizes e vetores utilizados no processo de solução tenham as dimensões estritamente necessárias e que não seja necessária a utilização de artifícios computacionais extras no processo de solução, melhorando a robustez numérica e o desempenho computacional.

Devido a grande flexibilidade obtida com o MICN ele pode ser utilizado desde para análises multifásicas desbalanceadas até sua utilização (sem alterações nos códigos do programa) para formulações puramente monofásicas, ou uma combinação de características, ou seja, pode simular quaisquer sistemas, reduzindo-se consideravelmente os custos de manutenção, de desenvolvimento e testes do programa computacional.

Definiu-se um novo tipo de equacionamento para vários equipamentos do sistema, aproveitando alguns modelos que já existiam, adaptando-os e melhorando-os, e desenvolveu-se novos modelos para alguns equipamentos, considerando características mais detalhadas, como por exemplo, a possibilidade de representar quaisquer aterramentos na modelagem de máquinas (correspondentes em metodologias convencionais a barras "PV" e "V $\theta$ "), o que não pode ser feito em muitos métodos.

Resultados obtidos com a metodologia proposta mostram que o MICN é bastante eficiente e robusto computacionalmente, necessitando de poucas iterações para solucionar os sistemas elétricos. Verifica-se nas simulações que o MICN permite representar e estudar diversos aspectos dos sistemas multifásicos cuja análise seria impossível de se realizar utilizando apenas ferramentas monofásicas ou até trifásicas.

O MICN é uma metodologia simples, eficiente e robusta, e não sofre das limitações apresentadas por vários métodos publicados na literatura. Sua habilidade em modelar precisamente muitos equipamentos típicos encontrados em sistemas reais é uma contribuição deste trabalho de pesquisa. Pode ser utilizado para modelar componentes com qualquer número de condutores, em muitas configurações, e é especialmente útil para resolver sistemas com controles, muito malhados ou de grande porte, e principalmente para sistemas de distribuição desequilibrados, quando a representação ou não dos detalhes pode fazer muita diferença nos resultados. As boas características de convergência, aliadas a possibilidade de grande detalhamento dos sistemas, sugerem que o MICN pode ser bastante útil em estudos de inclusão de geração distribuída e na análise de seus impactos em sistemas elétricos.

Considera-se então o MICN como sendo um método bastante geral podendo ser utilizado na análise de sistemas equilibrados ou desequilibrados, radiais ou reticulados, com cargas ou ramais monofásicos, bifásicos, trifásicos, multifásicos, com componentes com $n$ condutores, podendo ser utilizado em sistemas de transmissão, subtransmissão, distribuição e industriais, inclusive sistemas de grande porte. 


\section{REFERÊNCIAS}

Anderson, P. M. (1995). Analysis of Faulted Power Systems. IEEE Press Power Systems Engineering Series, New York, Nova York.

Araujo, L. R. (2000). Técnicas de Programação Esparsa Utilizando Modelagem Orientada a Objetos. Tese de M.Sc., Universidade Federal de Juiz de Fora, Juiz de Fora, MG, Brasil.

Baran, M. E. e Wu, F. F. (1989). Optimal Sizing of Capacitor Placed on a Radial Distribution System, IEEE Transactions on Power Delivery, v. 4, n. 1, pp. 725 - 734, Janeiro.

Castro, C. A. e Braz, L. M. C. (1997). Uma nova abordagem para a solução do problema de fluxo de carga pelo método de Newton com otimização de passo. Revista Brasileira de Controle \& Automação (SBA), Brasil, vol. 8, no. 3, pp. 121-133.

Céspedes, G. (1990). New Method for the Analysis of Distribution Networks, IEEE Transactions on Power Delivery, v. 5, n. 1, pp. 391 - 396, Janeiro.

Chen, T. H. e Yang, W. C. (2001). Analysis of multigrounded four-wire distribution systems considering the neutral grounding. IEEE Transactions on Power Delivery, vol. 16, n. 4, pp. 710-717, Outubro.

Cheng, C. S. e Shirmohammadi, D. (1995). A Three-Phase Power Flow Method for Real-Time Distribution System Analysis, IEEE Transactions on Power Systems, vol. 10, no. 2, pp. 671-679, Maio.

Ciric, R. M.; Feltrin, A. P. e Ochoa L. F. (2003). Power Flow in Four-Wire Distribution Networks - General Approach, IEEE Transactions on Power Systems, vol. 18, no.4, pp. 1283-1290, Novembro.

Costa, V. M.; Martins, N. e Pereira, J. L. R. (1999). Developments in the Newton Rapshon Power Flow Formulation Based on Current Injections. IEEE Transactions on Power Systems, v.14, n. 4, pp. 1320-1336, Novembro.

Dugan, R. C. (2004). Experiences with the Center-tapped Wye-Delta Transformer Test Case, 2004 IEEE PES General Meeting Conference Proceedings, Denver, Junho.

Elgerd, O. L. (1977). Introdução à Teoria de Sistemas de Energia Elétrica. Editora McGrawHill do Brasil, São Paulo, São Paulo.

Garcia, P. A. N.; Pereira, J. L. R. e Carneiro Jr, S. (2001a). Fluxo de Potência Trifásico por Injeção de Corrente -
Parte 1: Modelagem e Formulação Básica. Revista Brasileira de Controle \& Automação (SBA), Brasil, vol. 12, no. 3, pp. 178-187.

Garcia, P. A. N.; Pereira, J. L. R. e Carneiro Jr, S. (2001b). Fluxo de Potência Trifásico por Injeção de Corrente Parte 2: Controle e Dispositivos FACTS. Revista Brasileira de Controle \& Automação (SBA), Brasil, vol. 12, no. 3, pp. 188-196.

Gomes, F. V.; Vinagre, M. P.; Pereira, Z. L. R e Garcia, P. A. N. (2002). Fluxo de Carga em Sistemas que Apresentam Linhas de Potência Natural Elevada (LPNE). Revista Brasileira de Controle \& Automação (SBA), Brasil, vol. 13, no. 3, pp. 290-297.

Haffner, S.; Pereira, L. A. e Gasperin, L. V. (2008). Modelo Aproximado para Representação de Redes de Distribuição de Energia Elétrica, XVII Congresso Brasileiro de Automática (CBA), Juiz de Fora, Brasil.

Issicaba, D.; Coelho, J. e Alcântara M. V. P. (2008). Método de Soma das Potências com Rotações para Redes de Distribuição de Energia Elétrica, XVII Congresso Brasileiro de Automática (CBA), Juiz de Fora, Brasil.

Iwamoto, S. e Tamura, Y. (1981). A load flow calculation method for ill-conditioned power systems. IEEE Transactions on Power Apparatus and Systems, vol. PAS100, pp. 1736-1743.

Luo, G. X. e Semlyen, A. (1990). Efficient load flow for large weakly meshed networks. IEEE Transactions on Power Systems, vol. 5, pp. 1309-1316, Novembro.

Marinho, J. M. T. e Taranto, G. N. (2008). A Hybrid ThreePhase Single-Phase Power Flow Formulation, IEEE Transactions on Power System, vol. 23, no. 3, pp. 10631070, Agosto.

Penido, D. R. R.; Araujo, L. R.; Carneiro Jr., S.; Pereira J. L. R. e Garcia, P. A. N. (2008). Three-Phase Power Flow Based Four-Conductor Current Injection Method for Unbalanced Distribution Networks, IEEE Transactions on Power Systems, vol. 23, no. 2, pp. 494-503, Maio.

Kersting, W. H. (2008). A Three-phase Unbalanced Line Model with Grounded Neutrals through a Resistance, Panel Discussion on Stray Voltage Analysis Techniques, Proceedings of 2008 IEEE PES General Meeting, Pittsburg, PA, Julho.

[Online]. IEEE Test Feeders (2006), http: / / ewh. ieee. org/soc/pes/dsacom/testfeeders.html 